\title{
Teaching optics in the laboratory
}

\section{Christopher Dainty}

Christopher Dainty, "Teaching optics in the laboratory," Proc. SPIE 3190, Fifth International Topical Meeting on Education and Training in Optics, (8 December 1997); doi: 10.1117/12.294375

Event: Fifth International Topical Meeting on Education and Training in Optics, 1997, Delft, Netherlands 


\title{
Teaching Optics in the Laboratory
}

\author{
J C Dainty \\ The Blackett Laboratory, Imperial College \\ London SW7 2BZ ENGLAND
}

\begin{abstract}
In this paper, I outline the approach taken at The Blackett Laboratory (Physics Department) of Imperial College to the teaching of optics through laboratory work. The discussion includes both the undergraduate degree programmes (three year BSc and four year MSci) and the one year specialist MSc programme in Applied Optics.
\end{abstract}

Keywords: Education, Teaching

\section{INTRODUCTION}

The Blackett Laboratory (Physics Department) at Imperial College has the largest physics degree programme in the UK, with an annual intake of approximately 200 students for the three- and four-year courses, leading to the BSc (Bachelor of Science) and MSci (Master of Science) degrees respectively. The Department also has several specialist one-year postgraduate courses, including one in Applied Optics leading to the MSc (Master of Science) degree*. Before describing the teaching of optics through laboratory work, it is important to note a number of relevant local educational issues that apply in England ${ }^{\dagger}$ and may also apply elsewhere.

- In the last two years of school, typically at ages 17 and 18 , students study only 3 or 4 subjects to so-called Advanced Level: those intending to continue further study in the physical sciences typically do Mathematics (this can be spilt into two "A" levels, Pure Maths and Applied Maths), Physics and Chemistry. Most students entering Imperial College have very high grades in these subjects but because of declining average standards they do not have the depth of understanding that existed a decade or two ago, particularly in Mathematics.

- University education in England lasts for either three years or four years, i.e. students in physics, for example, leave with a BSc degree at age 21 or an MSci degree at age 22: even the latter course is shorter than those in many other European countries.

- Many University courses in the UK have a relatively small number of formal teaching hours per week, and indeed, it appears that the higher the ability of the students, the smaller the number of teaching hours. For example, at Oxford and Cambridge, the top two Universities for Physics in the UK, the students have $<15$ hours of formal teaching per week and a total of only 24 College weeks each year. At Imperial College, arguably a close third to Oxford and Cambridge, we provided $\approx 18$ formal teaching hours per week and a 30 week year, of which the last 4-5 weeks are taken up by Examinations. There are pros and cons of this low number of formal teaching hours.

- University physics courses in the UK stress physical principles as opposed to formal mathematical descriptions of physical phenomena.

- Traditionally, there is a very low "drop-out" rate of students in UK University courses. At Imperial College, for example, it amounts to a handful of students $(\approx 5)$ each year. The system of University funding means that the teaching income of the University is tied directly and precisely to the number of registered students; in approximate terms, the loss of 10-15 undergraduate students in one year would lead directly to the loss of one staff position in the following year. This of course means that there is a conflict of interest for the teaching staff, since the maintenance of high standards (requiring the removal of weaker students) could lead to the loss of their own job. On the more positive side, however, it is important to stress that this system means that a

${ }^{*}$ Note that the MSc refers to a post-graduate Master's degree, i.e. a second degree, whereas the abbreviation MSci refers to an undergraduate Master's degree, i.e. a first degree: the MSci degree is given the better name, MPhys, at a number of Universities.

${ }^{\dagger}$ In particular, Scotland has a different educational system. 
very great effort is made to ensure that every student reaches the potential of her or his ability, and that no one is left behind. In The Blackett Laboratory, for example, there is a very strong tutorial system: each student, for the first three years, sees a member of the academic staff once per week in a group of four students, usually to discuss the lecture courses and for problem solving. The personal attention paid to the needs of each student is a high priority and this is a great strength of a system that otherwise contains some inadequacies.

- Finally, most physics degree courses in the UK include a fairly large amount of practical laboratory work, typically 6 hours a week for three years. At Imperial College, in common with other Universities, senior staff (including full professors) are included amongst the demonstrators in the laboratory.

Turning to the teaching of optics in the Physics degree programmes at Imperial College, we have to face a few facts of life. First and foremost, we have to recognise that optics is a very small part of physics, and generally, in the undergraduate curriculum, is not in the same intellectual league as subjects like quantum mechanics, relativity or cosmology. I think it is very important to recognise this fact. Of course we should be enthusiastic for our own love of optics but equally we should appreciate that it is just a small part of a much bigger picture. Translating this to the requirements of the employers of our future students, there is an increasing emphasis placed by employers on multi-disciplinary skills and awareness.

Taking the three year BSc Physics degree as an example, approximately 300 hours of lectures are on compulsory topics (a further 150 hours are devoted to Option courses), and only 12 hours of these are directly on Optics, i.e. a student leaving our three year course may only spend $<3 \%$ of their lecture time directly on Optics! In reality, it is more likely that the average student does one more (optional) optics course during their undergraduate programme.

\section{UNDERGRADUATE OPTICS LABORATORY EXPERIMENTS}

In the circumstances we face in our Physics course - that is, with very little optics in the formal lectures - the rôle of optics experiments in the laboratory takes on a special significance. Traditionally, the main goals of laboratory experiments have been:

- To illustrate a topic that has been covered in lectures: the assumption in the past has been that the "theory" has been covered in lectures.

- To introduce students to the concepts of experimental design, errors, and interpretation of data: even now, one occasionally finds convoluted data gathering and analysis exercises in the laboratory that appear only to serve the purpose of teaching "errors", rather than physics.

In my view, these goals are inappropriate in our present context. The traditional goals have been replaced by new ones, in order of priority (written here specifically for the optics experiments but equally relevant for some other applied topics):

1. To teach optics through experimentation. Here the assumption is that the students have not done the "theory" in lectures.

2. To develop the confidence of students for actually doing physics, as opposed to thinking about doing physics.

3. To introduce ideas of experimental error, and the significance of data, in a natural way as the experimental goals dictate.

Some people find (1) rather radical: they believe that you first have to "understand" what you are doing by having lectures on the subject. I would argue that lectures rarely lead to proper understanding, and that learning-by-doing is an equally valid approach for many students. The second goal of developing confidence for practical work seems increasingly important as more and more of the learning process is centered on computers, simulation and virtual reality. The final goal of introducing ideas of experimental errors has to be done in the context of understanding the results of the experiment, not as an artificial exercise. 


\subsection{First Year Optics Cycle}

All first year $(\approx 19$ year-old) physics students do a four-week (24 hour) optics experiment cycle and for two-thirds of the students this comes before they have taken any lectures in optics. The cycle is therefore self-contained and relies only on elementary optics learnt at school. There are certain ground rules:

- All work, except the (short) "report" at the end of the experiment cycle has to be done during the laboratory sessions: we strongly discourage students from spending time in the evenings on writing up their laboratory records. This might at first sight seem a little perverse ... after all, if someone is keen to do more work, why should they be discouraged? The main reason is that there is a lot of other work to do in the evenings, such as digesting lectures notes and doing problem sheets, and six hours per week of laboratory is already one-third of the total contact time for each student.

- Everyone is required to keep a record of their work in a lab book. This is taken in after 1 week (6 hours) and 2 weeks (12 hours) and comments are written by the demonstrators to help the students improve their record-keeping but no marks are assigned at this stage. There is great emphasis placed on making a concise, accurate record of what actually happened in the experiment, not what should have happened.

- We promote active tuition by the laboratory demonstrators (about one demonstrator to 8-10 students). Students are continually interrupted and asked to explain what they are doing, and why, and results are discussed immediately after they are recorded (and repeated if necessary).

The optics cycle covers five topics:

1. Geometrical optics (6 hours)

2. Single and double slit interference and diffraction (9 hours)

3. The Michelson interferometer (3 hours)

4. Polarisation (3 hours)

5. Projects (3 hours)

Topics (1) and (2) form the core curriculum and are very prescriptive, with detailed instructions provided. In effect, students learn the subjects as they go along. Consider, as an example, the geometrical optics exercises: the first one is to find the focal length of a singlet lens (i) using the the autocollimation method and (ii) by determining several object and image distances and plotting the appropriate graph. Even these "simple" exercises have many potential pitfalls (e.g. where are the principal planes? What are principal planes?). Graphs are hand drawn and computer-drawn so that the values of slopes determined by eye and by least-squares fitting ${ }^{\ddagger}$. At the end of the day, the idea of the focal length, and how to find it, sinks in and I believe, like a lot of basic optical phenomena learnt in the laboratory, an understanding of it remains for many years.

Single and double slit diffraction and interference phenomena (topic 2) have been coverage by most first year students at school, so our emphasis here is to make photometric ${ }^{\S}$ measurements and compare them with theory. A laser diode is used as the source and photodiode as detector, with readings of the intensity of diffraction/interference patterns taken by hand from a digital voltmeter at fixed locations set by adjusting a micrometer screw (by hand). Of course we could automate this process, but what is the point of that? What does it teach? In this experiment, students rarely get a very good sinc-function for the single slit diffraction pattern, although it is certainly a plausible approximation: this immediately provokes a discussion with the demonstrator as to why theory and experiment are not in precise agreement.

Although topics (1) and (2) have detailed scripts, things get less prescriptive as the optics cycle progresses. The cycle ends with "projects" and here is the three-hour script in full :

${ }^{\ddagger}$ What is a least-squares fit? We have to give the essence of the concept without getting bogged down in details of algebra ...

$\S$ Photometric? That needs some explaining ... !

I Apart from safety warnings. 
Unlike the other the experiments, you are not given detailed instructions for the projects. Discuss the topics with the demonstrators. You have available the equipment used for the diffraction and interference experiments, and also the Michelson interferometer.

For each project, keep careful notes in your lab book. You will probably have to make several assumptions and simple calculations: make sure you keep a clear record of your work. Take a few minutes at the end of each project to review your lab book.

Each project should take 30 to 60 minutes, including keeping a record in your lab book.

\section{PROJECT A: Optical Disc}

In envelope $\mathbf{A}$ is (half of) a compact disc. Determine the information capacity of this disc in Gigabytes.

\section{PROJECT B: Thin Film Thickness}

In envelope $\mathbf{B}$ is a microscope slide with a thin film of magnesium fluoride on it (and an uncoated slide). Using the Michelson interferometer to determine the film thickness. The refractive index of magnesium fluoride is 1.38 .

\section{PROJECT C: Blood Cell Diameter}

In envelope $\mathbf{C}$ is a slide of dried blood (it is quite safe). Determine the diameter of the red blood cells on this slide.

In our experience, students need little prompting to do these experiments: they simply have to apply what they have learnt in previous weeks in the lab. So there is a progression, from very little knowledge or understanding to sufficient knowledge to actually do something useful.

There are, of course, many unsatisfactory aspects of the first year optics cycle. One is that we use the "real is positive" sign convention, leading to a form of the conjugate distance equation

$$
\frac{1}{p}+\frac{1}{q}=\frac{1}{f}
$$

which no optical engineer would ever use. We use Eq.(1) because that is the equation used by virtually all Physics textbooks. Another non-trivial problem is that the experiments have to be carried out in a darkened room, leading to fatigue (this could be eased by installing air-conditioning). A third problem is that the cycle is perhaps too intense, i.e we are trying to cover too much material.

\subsection{Second Year Diffraction/Holography Experiment}

By the second year, students are a little more street-wise and mature. The lecture course in the second year are tough: virtually all of them are on basic physics and we offer only one option course out of a total of 7 (large) courses. So an extra effort is made in the second year laboratory to be "user-friendly" and a more relaxed atmosphere is engendered. But we still want to teach optics through laboratory experiments, and to make the ideas stick in students' minds (none of the core second year lecture courses are on optics).

There are, in fact, several experiments involving optics in the second year physics lab but I will concentrate on only one of them, a 27 hour experiment itself split into two parts: (i) Fourier optics and diffraction and (ii) holography. The script is far less detailed than in the first year (11 pages compared to 27 in year one). The ratio of students to demonstrators is still about 10:1.

The holography part of the experiment (12-15 hours) is fun and for many years has been the most popular part of the whole second year lab. Using a $0.9 \times 1.2 \mathrm{~m}$ optical breadboard and magnetically mounted components, several different types of hologram are made, the most popular, not surprisingly, being those that are viewable in white light. I am not quite sure whether anything substantial is learnt here: just as taking a photograph does not mean that you understand the science of the photographic process, so taking a hologram does not really guarantee much understanding of holography. The one scientific part of this experiment is the construction of a holographic lens: this is then used at a range of object and image distances to verify the conjugate distance equation (Eq. 1 above). This brings home the fact that holography has potential applications other than "pretty pictures", and usually 
the discussion with the demonstrators turns to head-up displays, compact disc read heads, supermarket bar-code scanners and other applications of holographic optical elements.

In contrast to holography, the Fourier optics and diffraction part of the experiment (12-15 hours) is serious stuff. The goal here is to use optics to teach Fourier transforms, and to provide some glimpse as to why the Fourier interpretation of far-field diffraction is a useful one. As regards the physics of diffraction, this experiment does not go beyond the first year experiment mentioned in Section 2.1. Most of the main properties of Fourier transforms are illustrated, and, as with the rest of the experiment, no prior knowledge of Fourier transforms is required or expected. In fact, a course on Fourier transforms taught by a mathematician would probably be a handicap!

\section{LABORATORY EXPERIMENTS IN THE APPLIED OPTICS MSC COURSE}

The Applied Optics MSc Course is a specialist one-year postgraduate course, typically with 10-15 students who have a first degree in physics. Most of these students have done only a couple of optics courses in their first degree (i.e. $<10 \%$ of their undergraduate studies) and their ability and confidence in the laboratory work needs to be developed before they can carry out the tasks required of them by industry or in research. One of the reasons for this is that undergraduate laboratory experiments tend to be highly organised and regimented: to give a simple example, the apparatus provided for an experiment tends to be provided and laid out at the start of the experiment, whereas in real life the first task is to design the experiment and then procure the necessary equipment. Another feature of undergraduate experiments is that a detailed script is usually provided, whereas, of course, there is no script in real problem-solving.

There are approximately 174 hours of laboratory work in the MSc course divided into three principal activities, as given below. This laboratory work constitutes approximately $25 \%$ of the overall grade for the MSc degree (compared to $\approx 40 \%$ for all the lecture courses), which is a much higher percentage than for undergraduate courses. If there is one main goal of the laboratory in the MSc course it is to develop the confidence and ability to specify, design, build and align optical systems.

1. Demonstration Experiments. (42 hours) We start off with a series of 14 three-hour (one afternoon) experiments, each designed to introduce a single idea or concept. Examples include

- Critical and Köhler illumination

- Interpretation of Twyman-Green interferometer fringes

- Transmittance characteristics of colour filters

- Polarisation

- Optical fibre modes

- Star test

- Laser speckle

Lab books are taken after every second experiment and marked and returned for the next experiment is started (i.e. before the start of the next lab day) and the marking starts off in a very tough fashion, with the lab covered in constructive criticism by the demonstartors (academic staff). The whole experience is quite a shock for some of the students: for some of them it is the first time that their lab books have been mercilessly criticised, and the cycle of 14 experiments leaves both staff and students quite exhausted. At the end of this period we all deserve the "welcome party" for the new MSc class!

2. Standard Experiments. (72 hours) These follow the more traditional pattern, and consist of six 12-hour (i.e. one week) experiments that allow more in depth coverage of different topics, mainly in classical optics: currently these include:

- The Twyman-Green and Fizeau interferometers

- Holographic optical elements

- Laser modes 
- The star test

- Photon counting statistics

The emphasis is on a detailed understanding rather than superficial "knob-turning". For example, in the holography experiment, a $\mathrm{HOE}$ is made and the conjugate distance equation, angular equation and aberrations are studied.

3. System Design Project. (60 hours) The final part of the MSc laboratory cycle is to design, build and test a prototype of a simple instrument, concentrating on the optical engineering and the principal of the signal analysis. Examples of projects completed include:

- Bar code scanner

- Autofocus device

- Surface profiler

- Laser velocimeter

- Position sensor

The instructions are limited to a 200-word description of purpose of the instrument and therefore starts usually with a literature survey of possible ways of solving the problem. One of these has to be selected, and a set of

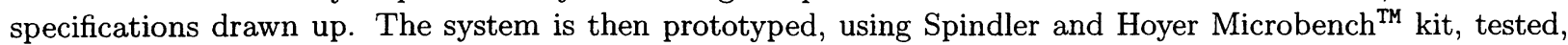
and its performance compared to the specification. A short report $(<20 \mathrm{pp})$ is written (including an estimate of the cost of making the prototype), within the 60-hour time limit.

Our hope is that by the end of the laboratory cycle, the students have both the ability and confidence to design, construct, test or use an optical system.

\section{CONCLUSIONS}

- The lack of formal optics lectures in our undergraduate physics degree programme has been balanced by the extensive teaching of optics through laboratory experiments.

- At the specialist postgraduate level in the one-year Applied Optics MSc course, the laboratory is used mainly to develop skills and confidence for a career in industry or in research.

\section{ACKNOWLEDGEMENTS}

The opinions discussed here are my personal ones, and some of them have found their way into the optics laboratory experiments in the Blackett Laboratory. Many staff and students have contributed to these experiments, and particular acknowledgments are due to Dr M J Damzen for his input to the undergraduate experiments and Professor R W Smith, Dr A A D Cañas, F Reavell and Dr K Weir in the MSc Laboratory over a number of years. 